\title{
Online Measurement of the Vasodilation of Peripheral Arteries on Ultrasound Images
}

\author{
E Bianchini ${ }^{1}$, R Andrei, F Faita ${ }^{2}$, V Gemignani $^{2}$, Y Plantinga ${ }^{1}$, M Demi $^{2,3}$ \\ ${ }^{1}$ Department of Internal Medicine, University of Pisa, Pisa, Italy \\ ${ }^{2}$ CNR Institute of Clinical Physiology, Pisa, Italy \\ ${ }^{3}$ Esaote SpA, Florence, Italy
}

\begin{abstract}
The online measurement of the vasodilation of a peripheral artery is useful in many clinical applications. Moreover, ultrasound imaging is recommended because of the advantages of this technique such as non-invasivity and feasibility. However, an algorithm with a subpixel resolution is required to obtain a useful plot of the vasodilation when using ultrasound images since the temporal changes of the vascular diameter on these images are usually not greater than 1-2 pixels. The algorithm we propose in this paper is based on the first absolute central moment, a mathematical operator which is used as an edge detector. The performances of the algorithm are analysed both on test discontinuities and on the online analysis of the brachial artery in in-vivo studies.
\end{abstract}

\section{Introduction}

In clinical applications, such as endothelial function assessment [1] and studies of arterial compliance or stiffness [2], the temporal changes of the diameter of a peripheral artery are highlighted on ultrasound images by movements of the vascular walls, which are usually not greater than 1-2 pixels. Therefore, to obtain a useful plot of the vasodilation, an algorithm which is able to locate edges on the image plane with a subpixel resolution is required. With this work two subpixel edge operators were implemented and compared: the normalized gradient of Gaussian (NGoG) and the mass center of the gray level variability. First, we derived a simple model of the gray level discontinuity provided by the vessel borders from a significant set of B-mode images of a brachial artery. The model, which is obtained from the sum of a smoothstep discontinuity and a Gaussian function, is subsequently used to analyse the performances of the two operators. In this way, the best configurations of the two edge operators were defined by varying the work conditions. Subsequently, we generated a set of ultrasound synthetic images by using the simulation software Field II [3] to verify the resolution of the two edge operators in ultrasound applications. A sequence of 100 realistic images of an artery, with a diameter ranging from $3.9 \mathrm{~mm}$ to $4 \mathrm{~mm}$, was thus obtained with steps of $0.01 \mathrm{~mm}$ and with a resolution of 13 pixels $/ \mathrm{mm}$. In this way, a real ultrasound exam of a brachial artery was simulated, during which the temporal changes of its diameter generated movements of the vascular walls which were less than 2 pixels. The analysis of this image sequence confirmed the capacity of the two edge detectors to detect diameter variations in the order of 0.1 pixels. Finally, the two algorithms were used for the online analysis of the brachial artery in in-vivo studies. Here again, variations of the vascular diameter in the order of 0.1 pixels were detected, thus providing a fine description of the vasodilation.

\section{The mass center of the gray level variability}

The first absolute central moment is a statistical filter which measures the variability of the gray levels of the image with respect to the local mean. Let $f(x, y)$ be the gray level map of an image, and let $g\left(x, y, \sigma_{i}\right)$ be normalized Gaussian weight functions. The following relationship is used to compute the first absolute central moment at a point $\mathbf{p}$ of coordinates $x, y$.

$$
e(\mathbf{p})=\iint_{h}\left|f(\mathbf{p}+\boldsymbol{\tau})-f(\mathbf{p}) \otimes g\left(\mathbf{p}, \sigma_{1}\right)\right| g\left(\boldsymbol{\tau}, \sigma_{3}\right) d \tau_{x} d \tau_{y}
$$

Moreover, the mass center $\mathbf{b}$ of the gray level variability associated to the first absolute central moment can be computed at $\mathbf{p}$ :

$$
b(\mathbf{p})=\left\{\begin{array}{cc}
\frac{1}{e(\mathbf{p})} \iint_{2}\left|f(\mathbf{p}+\boldsymbol{\tau})-f(\mathbf{p}) \otimes g\left(\mathbf{p}, \sigma_{1}\right)\right| \boldsymbol{\tau} g\left(\boldsymbol{\tau}, \sigma_{3}\right) d \tau_{x} d \tau_{y} & e(\mathbf{p}) \neq 0 \\
0 & e(\mathbf{p})=0
\end{array}\right.
$$

In [4] it is shown that if configurations of eq. (2) with $\sigma_{l}=\sigma_{3} / \pi$ are used then vector $\mathbf{b}$ always locates a point $\mathbf{p}^{\prime}$ which is closer to the nearest discontinuity than $\mathbf{p}$, 
independently of the distance between $\mathbf{p}$ and the discontinuity. Therefore, given the points $\mathbf{p}_{\mathbf{i}}$ of an approximate starting contour, a discontinuity can be located by iteratively computing vector $\mathbf{b}$ at $\mathbf{p}_{\mathbf{i}}$ where the starting points for any new iteration are the points $\mathbf{p}_{\mathbf{i}}^{\prime}$ which are located with the previous iteration.

\section{The Normalized GoG}

When processing gray level discontinuities which have an ideal step shape the respective contours can be easily localized by using a mathematical operator which is known as the normalized gradient of Gaussian (NGoG). When given an approximate starting contour, the points of the final contour can be directly located by computing NGoG at the points of the starting contour alone [4, 5]. However, an iterative procedure based on NGoG can be also used to locate real discontinuities $[4,5]$. Let $f(x, y)$ be the image gray level map and $g(x, y, \sigma)$ be a Gaussian function with $\sigma^{2}>a$. A vector $\mathbf{u}$, which locates a set of points $\mathbf{p}_{\mathbf{i}}^{\mathbf{i}}$ that are closer to the discontinuity than $\mathbf{p}_{\mathbf{i}}$ is obtained when computing $\mathrm{NGoG}$ at the points $\mathbf{p}_{\mathbf{i}}$ of a starting contour.

$\mathbf{u}(x, y)=a \frac{\left\lfloor\left(f * g_{x}\right)\left(f * g_{x x}\right)+\left(f * g_{y}\right)\left(f * g_{x y}\right)\right\rfloor \mathbf{i}+\left\lfloor\left(f * g_{x}\right)\left(f * g_{x y}\right)+\left(f * g_{y}\right)\left(f * g_{y y}\right)\right\rfloor \mathbf{j}}{\left(f * g_{x}\right)^{2}+\left(f * g_{y}\right)^{2}}$

In this case also, given an approximate starting contour, the relative discontinuity can be located by iteratively computing vector $\mathbf{u}$.

\section{Experimental results}

A significant set of B-mode images of the brachial artery was acquired and a realistic model of the gray level discontinuity which is associated with the artery borders was derived. Fig.1 shows the profiles of the gray level discontinuities which are associated to the artery border.

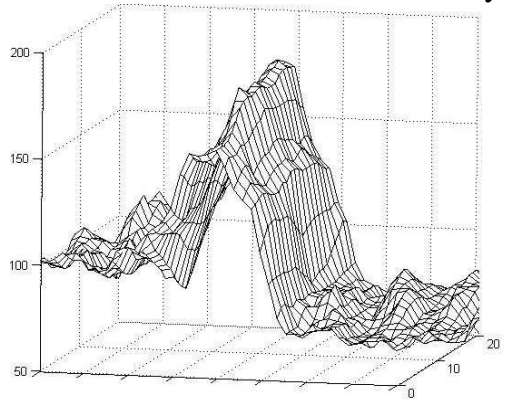

Figure 1. Profiles of the gray level discontinuities at the artery border

\subsection{Synthetic images}

A synthetic discontinuity similar to the real profile of the artery borders was obtained from the sum of a smoothstep discontinuity and a Gaussian function. An image (1280x1280 pixels) with two gray levels 120/100 i.u. (intensity unit) was used as a base. The step was then smoothed with a 2D Gaussian kernel with $\sigma_{2}=30$ pixels and a 1D Gaussian with $\sigma=30$ pixels was added. The maximum value of the obtained discontinuity model was equal to 180 i.u.. Fig. 2 shows the mathematical model of the gray level discontinuities which was obtained with the above procedure.

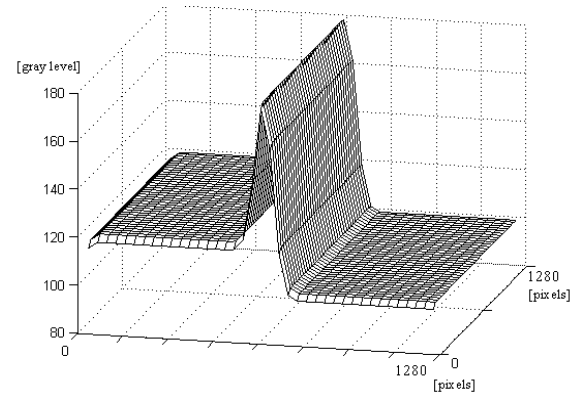

Figure 2. Mathematical model of the gray level discontinuity associated to the artery borders

In Fig. 3 the component $b_{x}$ of vector $\mathbf{b}$ is represented for different configurations of the operator which were obtained by varying the distance $\varepsilon$ between the starting point $\mathbf{p}$ and the point which represents the discontinuity (the top of the Gaussian function). Fig.3 shows that the point of convergence (the zero-crossing of the function $b_{x}(\varepsilon)$ ), of the iterative procedure based on the computation of vector $\mathbf{b}$, changes when varying the configuration of the mathematical operator.

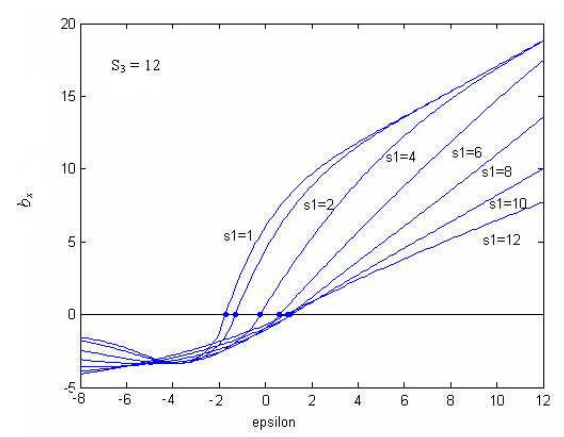

Figure 3. Component $b_{x}$ of vector $\mathbf{b}$, for different configurations of the operator, when varying the distance $\varepsilon$ between $\mathbf{p}$ and the discontinuity.

Subsequently, the algorithm based on the normalized gradient was applied to the discontinuity model with different apertures of the Gaussian function. In this case also, we observed that the point of convergence of the iterative procedure based on the computation of NGoG depends on the configuration of the operator as shown in Fig.4. 


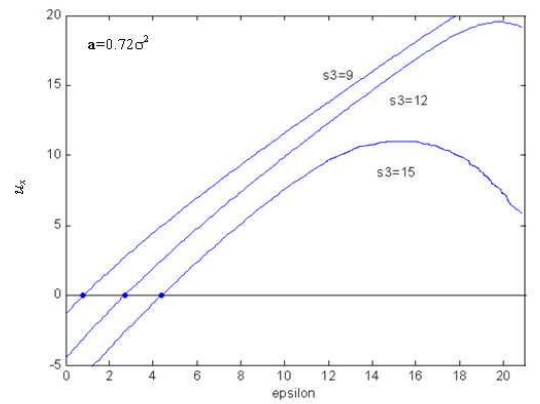

Figure 4. Component $u_{x}$ of vector $\mathbf{u}$, for different configurations of the operator, when varying the distance $\varepsilon$ between $\mathbf{p}$ and the discontinuity.

Finally, the mass center of the gray level variability and the normalized gradient were compared. Here the trends of the two edge detectors, when varying the distance of the starting point from the discontinuity, were found to be very similar. However, it is worth noting that the discontinuity is localized in two different points (Fig.5). Therefore, when discontinuities such as those of Fig. 2 are processed, the point of convergence depends both on the mathematical operator and on its configuration.

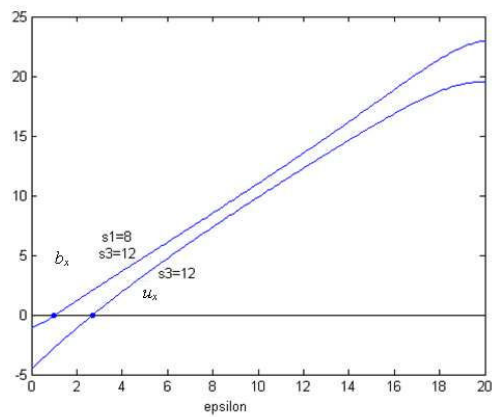

Figure 5. Component $u_{x}$ of vector $\mathbf{u}$ and $b_{x}$ of vector $\mathbf{b}$, when varying the distance $\varepsilon$ from the discontinuity

Subsequently, the two edge detectors were applied to more realistic synthetic images of the brachial artery obtained by using the software Field II. A 3D phantom was used to generate the images and the vessel was modeled as a cylinder positioned parallel to the probe. 100,000 scatterers were randomly distributed within the volume of the phantom and strong scatterers were placed to simulate boundaries, thus obtaining images which are very similar to the images obtained with standard echographic equipment. Scans were obtained by simulating a $13 \mathrm{MHz}$ linear array vascular probe with 192 elements spaced of $0.245 \mathrm{~mm}$. The images thus obtained were B-mode 256 gray levels with a resolution of 13 pixels/mm (Fig.6).

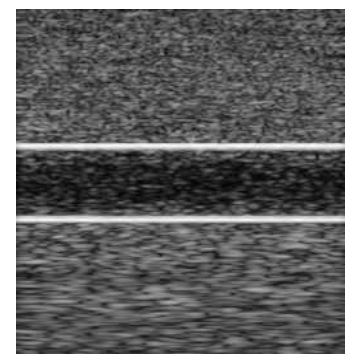

Figure 6. Image obtained with Field II.

To simulate a real ultrasound exam, a set of 100 images was synthesized. The sequence simulates five cardiac cycles where the diameter $d$ of the artery varies between $3.9 \mathrm{~mm}$ and $4.0 \mathrm{~mm}$. Since the resolution is 13 pixels $/ \mathrm{mm}$, the diameter variation in the image sequence is 1.3 pixels, ranging from 50.7 to 52.0 pixels. The sequence was analysed both with NGoG and the mass center of the gray level variability and the measurements of the diameter are represented in Fig.7.

The configuration $\sigma_{I} / \sigma_{3}=2 / \pi$ with $\sigma_{3}=4$ pixels was chosen for the mass center of the gray level variability and a value $a=0.72 \sigma^{2}$ with $\sigma=4$ pixels was chosen for NGoG. a)

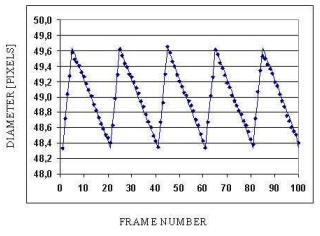

b)

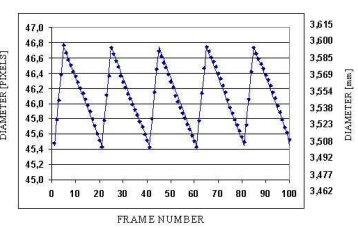

Figure 7. The figures show the diameter $d_{i}$ (continuous line) and its measure $\hat{d}_{i}$ (dotted line) minus the mean value of $\xi_{i}$, respectively, provided by the mass center of the gray level variability (a) and by NGoG (b).

Let $d_{i}$ be the diameter of the cylinder which is used to model the artery in the phantom and $\hat{d}_{i}$ be the diameter measured in the $\mathrm{i}^{\text {th }}$ image, the error is $\xi_{i}=\hat{d}_{i}-d_{i}$.

The statistics of the error were calculated for the two edge detectors. The mean and the standard deviation of the error are -5.2 pixels $(0.4 \mathrm{~mm})$ and 0.04 pixels $(0.003$ $\mathrm{mm})$, respectively, for $\mathrm{NGoG}$ and -2.3 pixels $(-0.18 \mathrm{~mm})$ and 0.03 pixels $(0.002 \mathrm{~mm})$, respectively, for the mass center of the gray level variability. Results show that both the measurements have a significant bias. Therefore, these results confirm that the location of these points depends on the algorithm and on the configuration of the mathematical operator. It is worth noting that the mean values of the errors obtained with the mass center of the 
gray level variability and with the NGoG are different and this is in line with the previous results. We have already seen in Fig.5 how the two operators, when applied to the discontinuity model, converge to two different points and how the distance between these two points is in the order of 2 pixels. Results confirm that the two algorithms give rise to two different bias levels even though the presence of a bias does not influence the assessment of the absolute diameter changes $\left(\Delta d_{i}=d_{i^{-}}\right.$ $\left.d_{i-1}\right)$. Moreover, the low standard deviation of the error substantiates the accuracy of the two algorithms when measuring the absolute diameter changes.

\subsection{Real ultrasound images}

Finally, the two algorithms were applied to B-mode images of longitudinal sections of brachial arteries in order to compute the diameter of the vessel in real-time. For this reason, the algorithms were implemented on a stand-alone video processing system based on a DSP board. Fig. 8 shows the plot of the diameter of a brachial artery which was obtained in an in-vivo examination when using the mass center of the gray level variability. The conditions were similar to those which we set with Field II $(13 \mathrm{MHz}$ linear array probe and an image resolution of $15.7 \mathrm{pixel} / \mathrm{mm}$ ). A sub-pixel precision of the measure was clearly obtained since the curve of the diameter change during the cardiac cycle is correctly traced even when the difference between the maximum and minimum value is in the order of one pixel. However, this implementation highlighted that $\mathrm{NGoG}$ is computationally twice more onerous than the mass center of the gray level variability and this is a critical point.

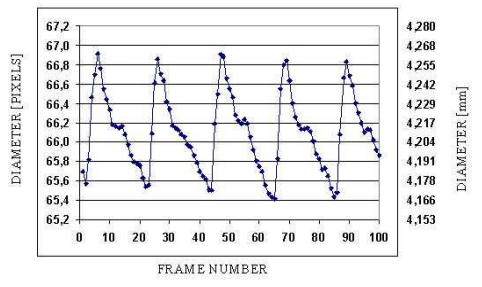

Figure8. Real-time in vivo analysis using the mass center of the gray level variability

\section{Discussions and conclusions}

In this work the subpixel edge detection properties of NGoG and the mass center of the gray level variability were analysed. These two operators were applied to a model of the gray level discontinuity which echographic imaging generates at vascular borders and it was highlighted that, for each operator, the point of convergence changes with the operator configuration. Moreover, in Fig.5, it is clearly shown that when both algorithms are applied to the same discontinuity they converge to two different points. Therefore, the location of this kind of discontinuity depends on the edge detector and on its particular configuration.

Then both algorithms were applied to a set of synthetic images of the brachial artery obtained with Field II by simulating working conditions similar to those which characterize a real vascular exam. This sequence reproduces five cardiac cycles where the difference between the maximum and minimum value of the diameter is in the order of one pixel. The results are affected by a bias since the location of the discontinuity depends on the edge operator and on its configuration. However, small variations of the diameter were detected and a very low standard deviation of the error was obtained for both operators.

Thus the operators were implemented on a stand alone video processing system and they both traced the curve of the diameter with high precision on real ultrasound images of the brachial artery. This is clearly shown for the mass center of the gray level variability in Fig.8 where small diameter variations in the order of 0.1 pixels are detected. However, the computational cost of the two edge detectors was compared and NGoG appeared about twice more onerous. Therefore, the mass center of the gray level variability should be used to measure vasodilation of the peripheral artery with subpixel precision in real-time analysis.

\section{References}

[1] Corretti MC et al. Guidelines for the ultrasound assessment of endothelial-dependent flow-mediated vasodilation of the brachial artery: a report of the International Brachial Artery Reactivity Task Force. J Am Coll Cardiol. 2002 Jan 16; 39(Pt 2):257-265.

[2] Budoff MJ, Flores F, Tsai J, Frandsen T, Yamamoto H, Takasu J. Measures of brachial artery distensibility in relation to coronary calcification. Am J Hyperten. 2003 May;16(5 Pt 1): 350-355.

[3] Jense JA. Field: A Program for Simulating Ultrasound Systems. Medical \& Biological Engineering \& Computing. 1996; 34 Suppl 1 (Pt 1 ):S351-353.

[4] Demi M. On the Gray-Level Central and Absolute Central Moments and the Mass Center of the Gray-Level Variability in Low Level Image Processing. Computer Vision and Image Understanding. 2005;.97(2):180-208.

[5] Fukunaga K, Hostetler LD. The estimation of the gradient of a density function with applications in pattern recognition. IEEE Trans. Info. Thy. 1975; IT-21( no.1): 3240.

Address for correspondence:

Bianchini Elisabetta

Institute of Clinical Physiology

Via Moruzzi, 156124 Pisa, Italy

betta@ifc.cnr.it 